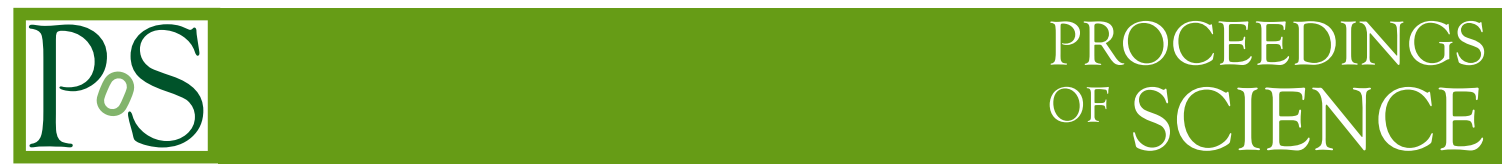

\title{
Optimal control methods for immunotheraphy
}

\author{
Benedetto Piccoli* \\ I.A.C.-C.N.R. \\ Viale del Policlinico 137 \\ 00161 Roma, Italy \\ E-mail: b.piccolieiac.rm.cnr.it
}

\section{Filippo Castiglione}

I.A.C.-C.N.R.

Viale del Policlinico 137

00161 Roma, Italy

E-mail: f.castiglionediac.cnr.it

We build a population dynamics model of the competition among immune system cells and a generic tumor cells. Then, we apply the theory of optimal control to find the optimal schedule of injection of autologous dendritic cells used as immunotherapeutic agent.

Control Systems: Theory, Numerics and Applications

30 March - 1 April 2005

Rome

${ }^{*}$ Speaker. 


\section{Background}

Mathematical models in biology are being used since Lotka (1925) and, independently, in 1926 Volterra formulated the predator-prey model of biological species. Today, by means of powerful computers the possibility to solve numerically complex mathematical formulations of biologicalmotivated problems is a routine task.

Computational and mathematical models are helping biologists to understand various aspects of the complex realm of living matter, from the beating of a heart to the molecular machinery underlying the cell-division cycle and cell movement.

A specific area of study is that of the immune system dynamics. Mammalian immune system can be considered one of the most complex systems nature has ever created. It is in charge to fight against all kind of potentially dangerous agent which break the anatomic barriers of the host organism [6]. The IS is composed by a variety of organs, cells and molecules acting in concert to achieve the basic functions of the IS, that is, recognition, response and memory.

When the immune system does not work properly the results is a disease. In the case of a tumor, the immune system should be able to detect the anomalous cells and kill them. Failure in this talk results in an uncontrolled growth of the tumor mass.

Immunotherapy is the "art" of stimulating the immune system to react against something specific. For example, for people which are allergic to bee sting, doctors repeat to inject small doses of venom until the immune system "changes" its way if reacting to the venom. Another example, which is relevant to us, is the immunotherapy applied to cancer. Of course, there are different way of stimulating the immune system. One of such way is to show to the IS pieces of the tumor telling him that they should be destroyed. The hope is that, once instructed, the immune system cells will be able to recognize the same pieces of tumor and destroy the cells bearing them.

Dendritic cells are perhaps the best so-called antigen-presenting-cells (APCs) in that their work consist in capturing the antigens (that is any potentially dangerous molecule they find around in the host body) and show them to other cells of the IS called effector cells. If the presented molecules are "judged" as dangerous, then the immune system mounts a specific response to eliminate the danger.

Dendritic cell transplantation is the practice of cultivating autologous dendritic cells (i.e., previously extracted from the same patient), together with characteristic molecules of the cancer cells (called tumor-associated-antigens or TAA) and then reinject them into the patient. The idea is that the immune system, confronted with such amount of tumor-antigen, starts to mount a response against it in place of a weak or completely absent response due to the inability of the TAA to induce a response because belonging to the self. As a side effect, it will eventually recognize the same molecules on tumor cells and kill them, actually eradicating the tumor.

There are many mathematical models of the immune system known in the literature [7] and a

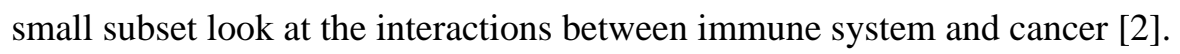

Our goal is to build a mathematical model to investigate the effect of tumor immunotherapy (and in particular Dendritic Cells Vaccine, DCV) for a generic solid a-vascular tumor. The obtained model is formed by five variables representing the cancer cells population and four key immune cells populations involved in the response of immune system to the cancer. The DCV represents 
the control part of the model and affects directly only the population of dendritic cells, while the tumor mass at the final time of the treatment period (optimization horizon) is the cost function.

After that, we apply the theory of optimal control to find the optimal protocol for the administration of DCVs loaded with TAA. The typical treatment consists of a finite number of DCV injections. Therefore we reduce to an optimization problem over a finite dimensional space, assuming that the vaccine administration follows always the same procedure.

Since the typical time scale, linked to cell duplication, of the model evolution is much bigger than that of the vaccine administration, we can use an approximation and optimal control tools to compute the gradient of the cost function with respect to the DCVs time schedule. More precisely, we consider a generalized setting for defining weakly differentiable control variations of a reference trajectory. Changes in the DCVs time schedule give rise to control variations that are weakly differentiable, hence we use the variational equation, along the reference trajectory, to compute the gradient of the cost function. The obtained approximation corresponds to consider each DCV injection as an impulse in the system evolution.

From computational point of view, our approach amounts to solve a linear system with a time varying matrix that is computed along the reference trajectory. Then any optimization algorithm, relying only on the gradient of the cost function, can be used to search minima of our problem.

Simulations are run with a C++ program via Runge-Kutta method to solve the differential equations. In principle, given a schedule, one should compute the corresponding reference trajectory and then solve the linear system given by the variational equation. However, this can be done in parallel to avoid memory allocation problems.

The results are quite satisfactory showing a decrease of the final value of the tumor mass with the use of a simple steepest descent method. However, when the number of injection is high the cost function depends in a stiff way from some of the injection times and the resulting optimization algorithm may present some numerical troubles. These were overcome via the use of a high order Runge-Kutta method both for the reference trajectory and the variational equation.

\section{The mathematical model}

The most popular mathematical models, in this and other fields, involve ordinary and partial differential equations (ODE) to represent reaction kinetics.

Ordinary Differential Equations (ODE) (or coupled-maps) are used to represent concentrations or populations of cells and/or molecules. The parameters represents kinetic constants.Partial differential equations (PDE) are often used to have a spatial representation of the diffusion of reagents.

\subsection{ODE modeling of tumor growth and dendritic cell presentation of tumor-associated-antigen}

The following ODE model is a very simple one. It is perhaps the only model specialized for autologous dendritic cell transfection therapy. We decided from the beginning to start with a simple representation of TAA-specific cells. We assume:

1. The time resolution is of one day. 
2. To use a monoclonal model in the first phase. Then look at Agur-Mazor policlonal model to think about a policlonal model which takes into account the immune response to two or more TAAs.

3. We will not include NK (natural killer) in our model. At least until there will be a good reason to do it. In general NK activity is non-specific so that a simple constant term in the equations would suffice.

4. No special reference to a specific tumor is necessary at a first stage of the math model development.

5. No geometry will be considered at this time.

6. The modelled tumor will be avascular.

7. Although it is clear that the competition between CTLs expressing different receptors important in this process. A recent paper by Vincenzo Cerundolo (Oxford, England) conclude that concurrent immunization with too many dominant epitopes will result in relative advantage for CTL specific for some epitopes at the expense of others. This aspect might be investigated when considering a policlonal model. At the first stage just tumor-specific cells will be taken into account, therefore no competition will be represented.

The model consists of few key immune cell populations, lymphocytes CD4 T helper cells and CD8 cytotoxic T cells, are modelled together with the population of cancer cell which refers to myelotic cells. Dendritic cells (the major antigen resenting cells in vertebrate immune systems) are the source of TAA and are introduced externally. The system is:

$$
\begin{aligned}
\frac{d H}{d t} & =a_{0}-b_{0} H+c_{0} D\left[d_{0} H\left(1-\frac{H}{f_{0}}\right)\right] \\
\frac{d C}{d t} & =a_{1}-b_{1} C+c_{1} I(M+D)\left[d_{1} C\left(1-\frac{C}{f_{1}}\right)\right] \\
\frac{d M}{d t} & =\left[d_{2} M\left(1-\frac{M}{f_{2}}\right)\right]-e_{2} M C \\
\frac{d D}{d t} & =-e_{3} D C \\
\frac{d I}{d t} & =a_{4} H D-c_{4} C I-e_{4} I
\end{aligned}
$$

Where $\mathbf{H}$ are the tumor-specific CD4 T helper cells, $\mathbf{C}$ are the tumor-specific CD8 T cells or CTLs citotoxic cell, $\mathbf{M}$ are the melanocytes (cancer cells) which expose the TAA, $\mathbf{D}$ are the mature dendritic cells (graft) which are loaded with the TAA (which expose tumor peptides on HLA molecule) and $\mathbf{I}$ is the IL-2 secreted by $\mathrm{H}$ and responsible for $\mathrm{T}$ cell growth.

Note that we decide to use the logistic growth proposed by Verhulst in $1836 R(x)=r\left(1-\frac{x}{K}\right)$ where the constant $K$ is the carrying capacity of the environment, which is usually determined by the available sustaining resources. $R(x)$ in this case represents the per capita birth rate, that is, it is dependent on $x$. 


\begin{tabular}{|c|l|l|}
\hline Name & description & value-units $(\star)$ \\
\hline$a_{0}$ & birth of CD4 T & $10^{-4} c d^{-1} \mathrm{~mm}^{-3}$ \\
$b_{0}$ & death of CD4 T & $0.02 d^{-1}$ \\
$c_{0}$ & max prolif of CD4 T & 10 \\
$d_{0}$ & $1 / 2$ satur const of CD4 T & $10^{-2} c^{-1} d^{-1} \mathrm{~mm}^{3}$ \\
$f_{0}$ & carrying capacity of CD4 T & $1 c m^{-3}$ \\
\hline$a_{1}$ & birth of CD8 T & $10^{-4} c d^{-1} m^{-3}$ \\
$b_{1}$ & death of CD8 T & $0.02 d^{-1}$ \\
$c_{1}$ & max prolif of CD8 T & 10 \\
$d_{1}$ & $1 / 2$ satur const of CD8 T & $10^{-2} c^{-1} d^{-1} \mathrm{~mm}^{3}$ \\
$f_{1}$ & carrying capacity of CD8 T & $1 c \mathrm{~mm}^{-3}$ \\
\hline$d_{2}$ & $1 / 2$ satur const of tumor & $0.02 d^{-1}$ \\
$e_{2}$ & killing by CD8 of tumor & $0.1 c^{-1} d^{-1} \mathrm{~mm}^{3}$ \\
$f_{2}$ & carrying capacity of tumor & $1 c \mathrm{~mm}^{-3}$ \\
\hline$e_{3}$ & CD8 T killing of DC & $0.1 c^{-1} d^{-1} \mathrm{~mm}^{3}$ \\
\hline$a_{4}$ & production by CD4 T & $10^{-2} c^{-1} d^{-1} \mathrm{~mm}^{3}$ \\
$c_{4}$ & IL-2 uptake by CD8 T & $10^{-7} c^{-1} d^{-1} \mathrm{~mm}^{3}$ \\
$e_{4}$ & degradation rate & $10^{-2} d^{-1} \mathrm{~mm}^{-3}$ \\
\hline
\end{tabular}

Table 1: Parameters of the model. Note that $e_{2}=e_{3}$ since both dendritic and cancer cells express the same TAA by assumption. $\star c$ stays for cells, and $d$ stays for days

The term $a_{0}-b_{0} H(t)$ in equation (2.2) represents the production by the bone marrow of a very small number of tumor specific cells. Although tumor antigens are very poor immunogenic, it is reasonable to think that very few cells able to recognize them circulates in our body. An equivalent term in equation (2.3) $a_{1}-b_{1} C(t)$ is given for tumor specific cytotoxic cells.

The term $c_{0} D(t)\left[d_{0} H\left(1-\frac{H}{f_{0}}\right)\right]$ in equation (2.2) represents the clone expansion of tumor specific helper cells upon presentation of tumor associated antigen by dendritic cells. Dendritic cells are injected into the host already loaded, hence presenting the tumor peptides bind both to MHC class I or II. A saturation term for the growth of helper cells is considered.

The term $c_{1} I(t)(M+D)\left[d_{1} C\left(1-\frac{C}{f_{1}}\right)\right]$ in equation (2.3) represents the clone expansion of tumor specific cytotoxic cells either by interaction with tumor cells or with dendritic cells. Since such clone expansion is possible only in presence of IL-2 which is secreted by Thelper cells upon recognition of dendritic loaded cells, no tumor specific response is possible without tumor antigen presentation by dendritic cells.

Myeloid cells grow (limited grow) $\left[d_{2} M\left(1-\frac{M}{f_{2}}\right)\right]$ in equation (2.4) and are killed by tumor specific cytotoxic cells $-e_{2} M(t) C(t)$.

Dendritic cells are killed by cytotoxic cells $-e_{3} D(t) C(t)$.

Interleukin IL-2 is produced by helper cells upon recognition of tumor loaded dendritic cells $a_{4} H(t) D(t)$ and is consumed by cytotoxic cells during clonal growth $-c_{4} C(t) I(t)$ (equation (2.5)). While the term $-e_{4} I(t)$ represents degradation of free interleukin.

The parameters are reported in table [. Values in the last column are (still) arbitrary, and are 
reported to remind the units.

After a suitable choice of the parameter values (without explicit reference to biological relevance) the model in equation (2.3) - equation (2.5) shows the following dynamics:

- In case of a lack of immune response the tumor will grow.

- If we administer a vaccine consisting of dendritic cells loaded with tumor-associated antigens, a specific cytotoxic immune response will be obtained. Figure 1 shows a reduction to almost zero of the tumor population. A tumor specific response with growth of cytotoxic cells and helper cells which recognize the tumor. CTL cells kill dendritic cells because they show the tumor peptides but also the tumor cells. IL- 2 is produced by helper cells upon contact with dendritic cells presenting tumor peptide together with MHC class II molecules. On the other hand, dendritic cells presenting tumor peptides on the class I MHC molecule are able to bind cytotoxic cell receptors and stimulate growht.
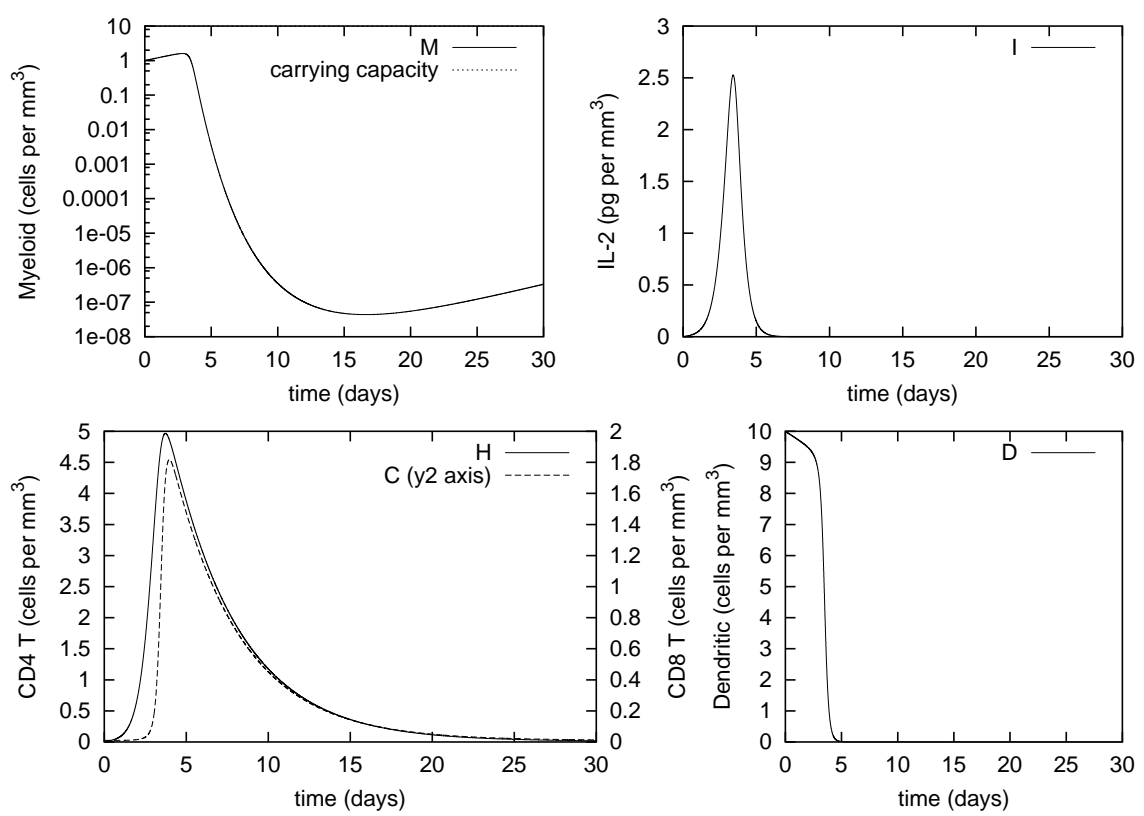

Figure 1: Introducing a population of dendritic cells which are loaded with TAA, one observe a tumor specific response with growth of cytotoxic cells and helper cells which recognize the tumor. CTL cells kill dendritic cells because they show the tumor peptides but also the tumor cells. IL-2 is produced by helper cells upon contact with dendritic cells presenting tumor peptide together with MHC class II molecules. On the other hand, dendritic cells presenting tumor peptides on the class I MHC molecule are able to bind cytotoxic cell receptors and stimulate growth.

Figure 7 shows the curves of tumor size versus time for different therapeutic regimen (DC injection doses). Larger doses correspond to a bigger reduction in tumor size. 


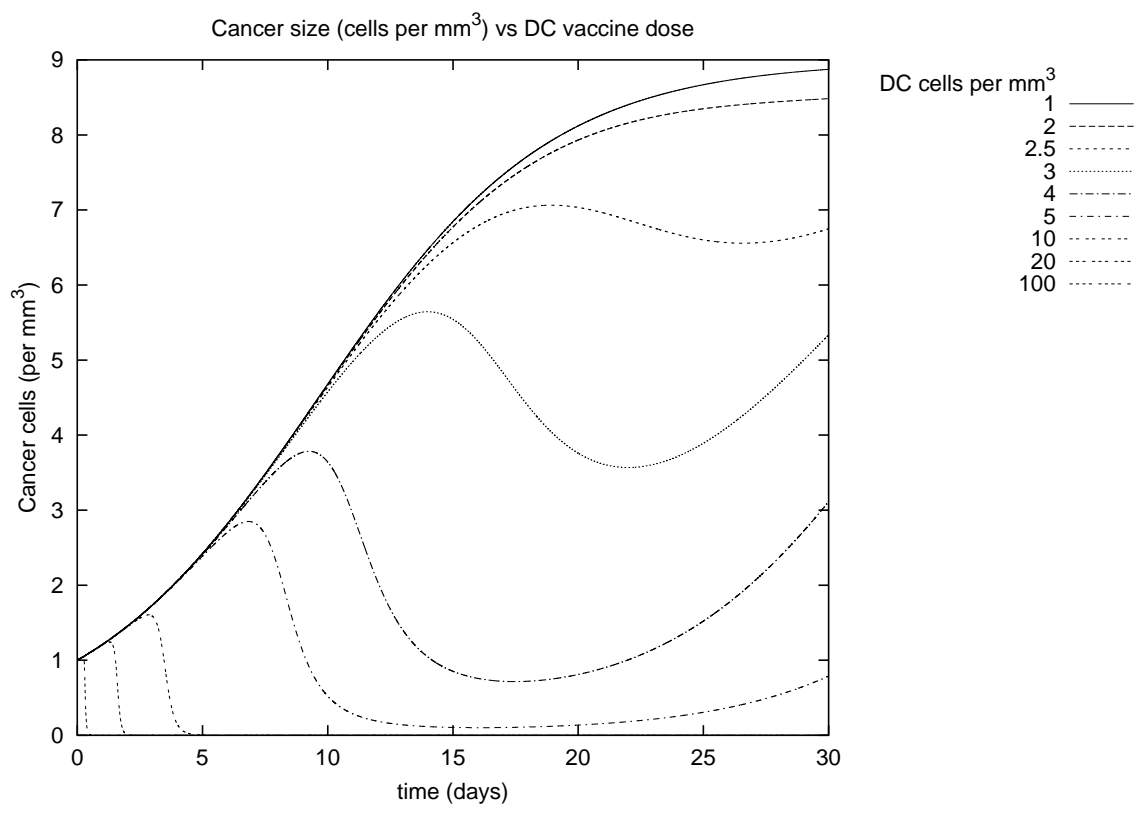

Figure 2: Cancer cells (per $\mathrm{mm}^{3}$ ) versus DC-vaccine dose (in cells injected per $\mathrm{mm}^{3}$ ).

\section{Optimal Control}

Consider a general control system:

$$
\frac{d x}{d t}=F(x, u)
$$

where $x \in R^{n}$ and $u \in U$ compact subset of $R^{m}$. We fix $T>0$ assume that $F$ is such that for every control function $u:[0, T] \rightarrow U$ and $x_{0} \in R^{n}$ there exists a unique solution $x(\cdot, u)$ satisfying $\frac{d x}{d t}(t, u)=F(x(t), u(t))$ a.e. on $[0, T]$ and $x(0, u)=x_{0}$. An optimal control problem in Mayer form is given by:

$$
\min _{u(\cdot) \in \mathcal{U}} \psi(x(T, u)), \quad x(0)=x_{0},
$$

where $\mathcal{u}$ is the class of admissible controls, $\psi$ the final cost, $T$ the terminal time and $x_{0}$ the initial condition. The aim is to find the control $u(\cdot) \in \mathcal{U}$ so that $\psi(x(T, u))$ is minimized.

The well known Pontryagin Maximum Principle (PMP), see [9], provides, under suitable conditions, a necessary condition for optimality in terms of a lift of the candidate optimal trajectory to the cotangent bundle. Such lift is a trajectory of a pseudo-Hamiltonian system. The proof of PMP relies on a special type of variations, called needle variations, of a reference trajectory. Given a candidate optimal control $u^{*}$ and corresponding trajectory $x^{*}$, a time $\tau$ of approximate continuity for $F\left(x^{*}(\cdot), u^{*}(\cdot)\right)$ and $\omega \in U$, a needle variation is a family of controls $u_{\varepsilon}$ obtained replacing $u^{*}$ with $\omega$ on the interval $[\tau-\varepsilon, \tau]$. A needle variation gives rise to a variation $v$ of the trajectory satis- 
fying the variational equation $\frac{d v}{d t}(t)=D_{x} F\left(x^{*}(t), u^{*}(t)\right) \cdot v(t)$ in classical sense (where $D_{x} F$ is the Jacobian of $F$ w.r.t. $x$ ), only after time $\tau$.

Recently, see [8, 10, 5], it was introduced a setting in which needle and other variations happen to be differentiable. First let $\mathcal{C}$ be the space of real continuous functions defined on $[0, T]$ and $\mathcal{M}$ its dual, the space of bounded Radon measures on $[0, T]$, see [ [

Definition 1. A parameterized family of controls $\left\{u_{\varepsilon}: u_{\varepsilon}:[0, T] \rightarrow U, \varepsilon \in[0, \bar{\varepsilon}]\right\}$ with corresponding trajectories $x^{\varepsilon}$ is weakly differentiable at $\varepsilon=0$ if

$$
w_{\varepsilon}(\cdot)=\frac{F\left(x_{0}(\cdot), u_{\varepsilon}(\cdot)\right)-F\left(x_{0}(\cdot), u_{0}(\cdot)\right)}{\varepsilon}
$$

converges for the weak ${ }^{*}$ topology of $\mathcal{M}$ to some measure $\mu \in \mathcal{D}$. In other words, for every continuous function $\phi \in \mathcal{C}$ the map $\varepsilon \mapsto \int \phi(t) w_{\varepsilon}(t) d t$ converges to $\int \phi d \mu$.

Given a weakly differentiable family $u_{\varepsilon}$ the corresponding trajectory variation $v$ satisfies:

$$
d v=D_{x} F\left(x_{0}, u_{0}\right) \cdot v d t+d \mu
$$

where now the equation is understood in integral sense. Thus for a needle variation we obtain:

$$
\begin{gathered}
d v=D_{x} F\left(x^{*}, u^{*}\right) \cdot v d t+ \\
\left(F\left(x^{*}(\tau), \omega\right)-F\left(x^{*}(\tau), u^{*}(\tau)\right)\right) d \delta_{\tau},
\end{gathered}
$$

where $\delta_{\tau}$ indicates a Dirac delta centered at $\tau$. Then one can prove PMP in the usual way.

More generally, consider a family of controls of type:

$$
u_{\varepsilon}=\sum_{i=0}^{N} u_{i}(t) \chi_{\left[t_{i}^{\varepsilon}, t_{i+1}^{\varepsilon}[\right.}
$$

where $u_{i}:[0, T] \rightarrow U$ is continuous, $0=t_{0}^{\varepsilon}<t_{1}^{\varepsilon}<\cdots<t_{N+1}^{\varepsilon}=T$, and $\chi$ is the indicator function. If $t_{i}^{\varepsilon}=t_{i}^{0}+\varepsilon+o(\varepsilon)$, then such a family is weakly differentiable and the variation $v$ of the corresponding trajectories $x_{\varepsilon}$ satisfies:

$$
\begin{gathered}
d v=D_{x} F\left(x_{0}, u_{0}\right) \cdot v d t+\sum_{i=1}^{N} d \delta_{t_{i}} . \\
\left(F\left(x_{0}\left(t_{i}\right), u_{i-1}\left(t_{i}\right)\right)-F\left(x_{0}\left(t_{i}\right), u_{i}\left(t_{i}\right)\right)\right) .
\end{gathered}
$$

\section{Optimal Control theory applied to Cancer Immunotherapy}

We now consider the DCV as a possible control on (2.1)-(2.5), hence we obtain the control system

$$
\frac{d x}{d t}=f(x)+u g(x)
$$

where $x=(H, C, M, D, I)$ represents the cells populations, the field $f$ is given by (2.1)-(2.5) and, since DCV acts only on Dendritic cells, we have $g(x)=e_{4}$ the fourth coordinate vector. Our cost is the final value of the tumor mass $M$. Thus we consider the optimal control problem:

$$
\min _{u(\cdot) \in \mathcal{U}} M(x(T, u(\cdot))), \quad x(0)=x_{0},
$$


where $T$ is the final time of the treatment period, $x_{0}$ is some fixed initial value of cells populations and the set $\mathcal{U}$ is still to be defined.

We consider a vaccine administration procedure described by a continuous control function

$$
\bar{u}:[0, \eta] \mapsto[0, \bar{V}]
$$

where $\bar{V}$ is the maximal vaccine quantity. The function $\bar{u}$ represents the value of injected Dendritic cells population as a function of time.

The most important time scale, in the evolution of the system (2.1)-(2.5), is given by the cellular duplication time, which is estimated about $1 / 3$ of a day. On the other side, the time duration of the vaccine administration is often of the order of one hour or less, hence very small compared to the natural time scale of (2.1). Therefore we assume that $\eta$ is very small.

Consider now a family of controls $u_{\varepsilon}$ corresponding to a single vaccine administration procedure that happens at some time $t_{\varepsilon}=\bar{t}+\varepsilon+o(\varepsilon)$. Then the family $u_{\varepsilon}$ is formed by control functions of type (3.3) and is weakly differentiable. Assuming that $\bar{u}$ is close to the constant $\bar{V}$, letting $\bar{x}$ correspond to the vaccination at time $\bar{t}$ and taking a first order expansion for the equation of $v$ we get:

$$
\begin{gathered}
-v((\bar{t}+\eta)+)=\int_{\bar{t}}^{\bar{t}+\eta} D_{x} f(\bar{x}(t)) \cdot \bar{V} e_{4} d t+o(\eta) \sim \\
\int_{\bar{t}}^{\bar{t}+\eta} D_{x} f\left(\bar{x}\left(\bar{t}+t \bar{V} e_{4}\right)\right) \cdot \bar{V} e_{4} d t= \\
f(\bar{x}(\bar{t})+V)-f(\bar{x}(\bar{t})),
\end{gathered}
$$

where for simplicity we set $V=\eta \bar{V} e_{4}$, that is the vector representing the Dendritic cell population actually inoculated during a vaccine administration. Finally we obtain the following:

Proposition 1. Let $u_{\varepsilon}$ be a family of controls corresponding to a single vaccine administration procedure at time $t_{\varepsilon}=\bar{t}+\varepsilon$ and $\bar{x}$ the trajectory corresponding to vaccination at time $\bar{t}$. Then the corresponding variation is approximated for $t \geq \bar{t}$ by the solution of:

$$
\left\{\begin{array}{l}
\frac{d v}{d t}(t)=D_{x} f(\bar{x}(t)) \cdot v(t) \\
v(\bar{t})=f(\bar{x}(\bar{t}))-f(\bar{x}(\bar{t})+V)
\end{array}\right.
$$

The clinical treatment of a patient via immunotherapy consists in a series of DCVs that are scheduled overtime range of some months. We then consider a control procedure that consists in $N$ vaccinations inoculated according to a schedule $S=\left\{t_{i}: i=0, \ldots, N, 0 \leq t_{1} \leq t_{2}-\eta<t_{2} \leq \cdots \leq\right.$ $\left.t_{N} \leq T-\eta\right\}$. Let $\mathcal{S}$ be the space of schedules, then for every $S \in \mathcal{S}$ we define $u(S)$ to be the corresponding control

$$
u_{S}(t)=\sum_{i=1}^{N} \bar{u}\left(t-t_{i}\right) \chi_{\left[t_{i}, t_{i}+\eta\right]} .
$$

The control $u_{S}$ corresponds to $N$ vaccine administration procedures that occur at times $t_{i}$. Finally we set

$$
\mathcal{U}=\left\{u_{S}: S \in \mathcal{S}\right\}
$$

and the optimal control problem (4.2) is now well defined: 
(P) Given the initial condition $x_{0}$ determine a schedule $S \in \mathcal{S}$ so that the trajectory $x_{S}$ of $\frac{d x}{d t}=$ $f(x(t))+u_{S}(t) g(x)$ attains the minimum of $M(x(T, u))$.

It is easy to notice that such optimal control problem is indeed a (finite dimensional) optimization problem. In fact the space $S$ can be clearly parameterized by a subset of $R^{N}$.

Moreover, thanks to Proposition 1, we can approximate this optimization problem considering the set of controls given by finite sums of delta functions centered at vaccination times of the schedule, thus considering $\eta=0$. The set $S$ is obviously compact and the function $S \mapsto M\left(x_{S}(T)\right)$ continuous, hence there exists a solution to $(P)$. Analogously to Proposition 1 , one can easily prove:

Proposition 2. For the problem $(P)$, up to an error of order $\eta$, we have:

$$
\frac{\partial M\left(x_{S}(T)\right)}{\partial t_{i}}=\nabla \psi\left(x_{S}(T)\right) \cdot v_{i}(T)=e_{4} \cdot v_{i}(T)
$$

where $v_{i}(\cdot)$ is the solution to (4.4) for $\bar{t}=t_{i}$ and $x_{0}=x_{S}$.

To solve numerically problem (P) we can use Proposition 2 and steepest descent or other optimization methods.

\section{Simulations.}

To run simulations we used a $\mathrm{C}++$ program consisting of the following iterative procedure.

Step 0 Fix the final time $T$, the number $N$ of vaccine administrations, the (vector) value $V$ of vaccine quantity, an initial value $x_{0}$ of cells populations and an initial schedule $S_{0}$.

Step 1 Solve the system (2.1)-(2.5) with initial value $x_{0}$ via Runge-Kutta integrator generating an approximation of the trajectory $x_{S}$. At the same time solve the variational equation $\frac{d v_{i}}{d t}=$ $D_{x} f\left(x_{S}\right) \cdot v_{i}$ with initial condition $v_{i}\left(t_{i}\right)=f\left(x_{S}\left(t_{i}\right)+V\right)-f\left(x_{S}\left(t_{i}\right)\right)$ for $i=1, \ldots, N$.

Step 2 Compute $\partial M\left(x_{S}(T)\right) / \partial t_{i}$ via Proposition 2 .

Step 3 Update the schedule according to steepest descent, i.e. add to $t_{i}$ the value $h \cdot \partial M\left(x_{S}(T)\right) / \partial t_{i}$ for some small parameter $h$. GOTO Step 1 .

Remark 1. Notice that in Step 1 we compute at the same time the solution to (2.1)-(2.5) and the variational equations (4.4), this allows to spare memory also in view of more complex models.

We consider the case of a time horizon $T$ of six months, $N=10$ DCVs injections. The initial value of the tumor is 0.1 , the $H$ and $C$ levels are set to equilibrium, while $I$ and $D$ are set to zero. This means that there is no specific response at initial time of the immune system. If no vaccination occur the tumor would tend to equilibrium value as explained in the model description. The vaccine quantity $V$ is set to 0.25 , cfr. with the effect on tumor level shown in Figure \&.

We run some simulations with initial schedule $S_{0}$ chosen randomly and 200 iterations. In Figure 3 we represent the outcome of final cancer cells population during a run of the program. Notice that the final value of the tumor decreased consistently from 0.35 of the first optimization 

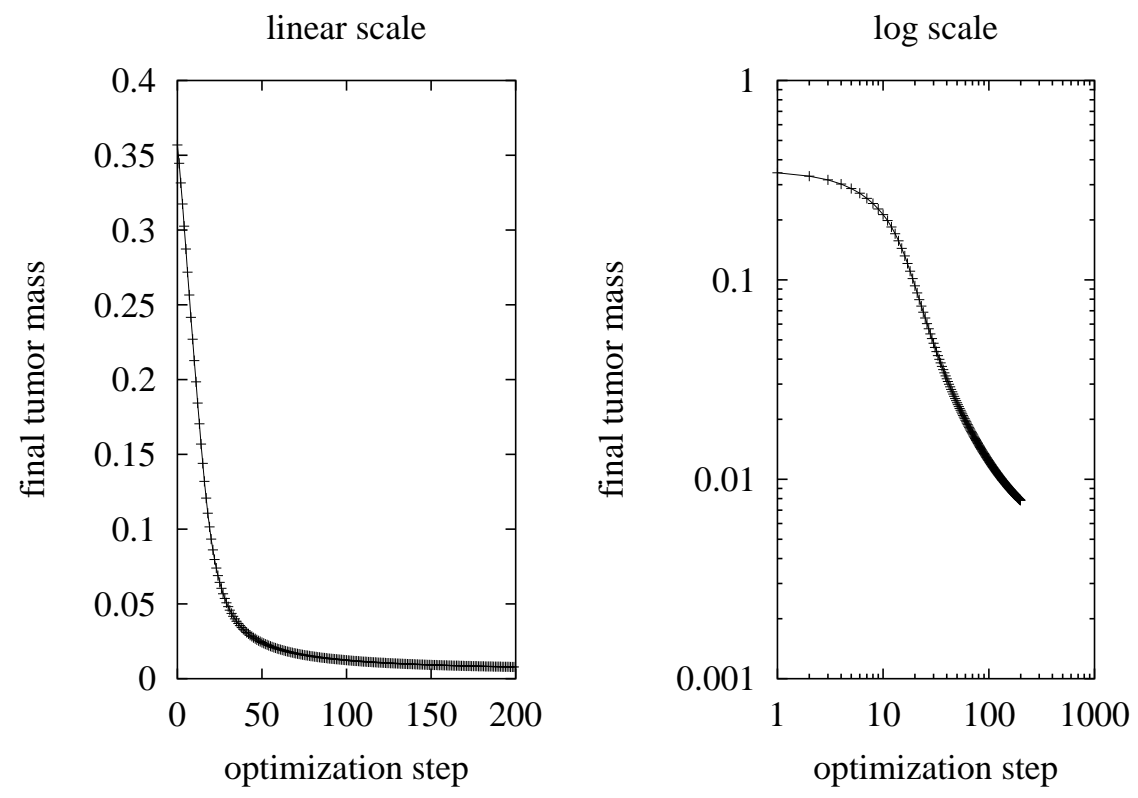

Figure 3: This figure shows the final value of the tumor (linear and logarithmic scale) for each optimization step.

step to an almost zero level, which means essentially extinction of the tumor. Unfortunately some run presents some numerical problems due to the stiffness of the system. In fact the final value of the tumor mass may be quite sensible with respect to some vaccination times.

In Figure $\bigoplus$ it is shown the evolution of the schedule during the iterations of the program. The number of iteration is plotted on the first axis, while in the second axis the vaccine administration times are depicted. The time value is represented in hours (4320 over six months). We notice that, as expected, the cost function is more sensitive with respect to late vaccinations. This is consequence of the fact that the effect of a DCV expires in time, again cfr. Figure 2. Therefore the optimization procedure mostly changes the last times of the schedule. In this precise example the first four vaccination times are not at all changed during the optimization procedure. The last two vary most rapidly and essentially collapse in a unique double vaccination since the beginning. The optimization is anyway far from trivial, for example the variation of vaccination times is not monotone as for numbers 5,9 and 10 .

To contrast the effect of pushing vaccination times to the end one should add a constraint or cost on the maximal value of the tumor during the whole time horizon. On one side this is natural for the model, on the other side the optimal control problem thus obtained is much more difficult to be treated.

In last Figure 5 the evolution of all variables for the first iteration is illustrated. As we noticed above, for the chosen values, the tumor is highly affected by the presence of the $\mathrm{H}$ (tumor specific 


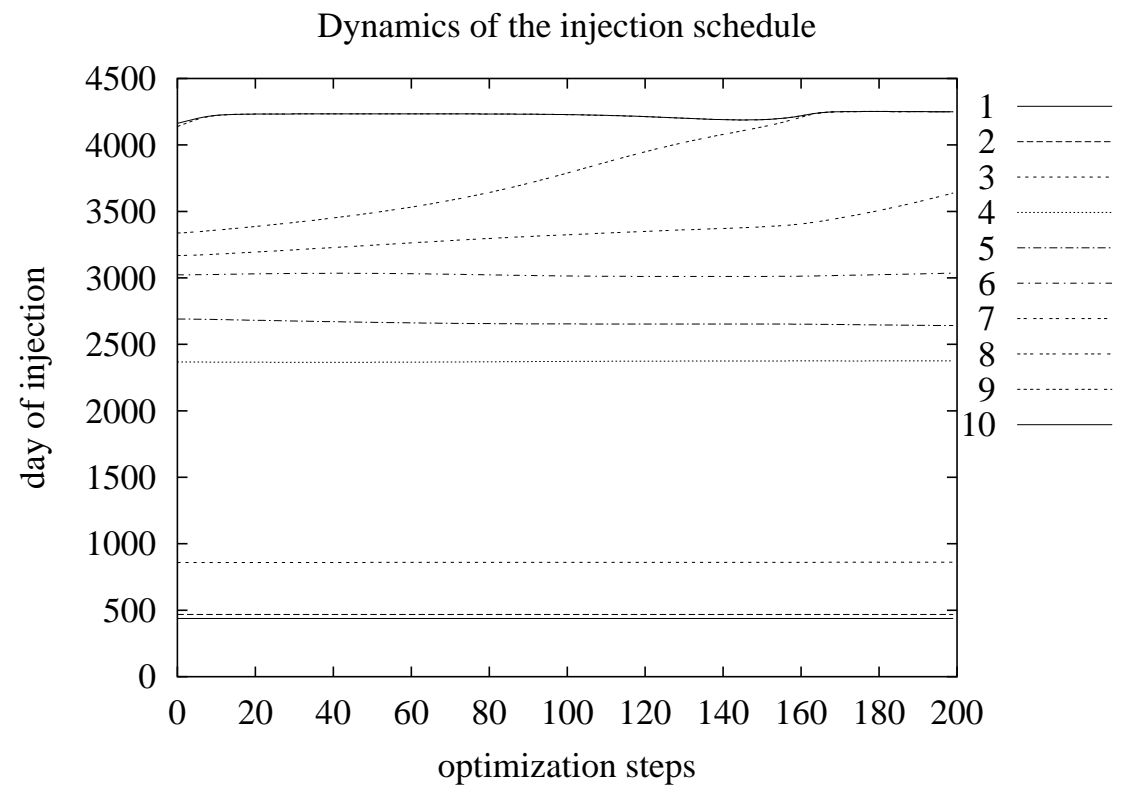

Figure 4: We plot here the injection schedule versus time. The initial schedule (or protocol) is chosen at random. The dynamics shows non trivial aggregation of injections at the beginning and at about half of the period.

$\mathrm{T}$ helper) cells and the $\mathrm{C}$ (tumor specific citotoxic) cells. The decay of these cells is quite rapid and even more is that of $D$ (Dendritic) and I (Interleukin). Let us remark again that the value of the tumor is not under control for the whole time horizon.

\section{Conclusions.}

We built a mathematical model for the immunotherapy of a generic cancer via Dendritic Cell Vaccines (DCVs). The obtained system represents the cancer cells population and the main immune cells populations involved in the response of the immune system. The control is given by the schedule of injections of Dendritic cells via DCVs and The dynamics of the not controlled systems is discussed in detail to test the response of immune system to cancer cells and the effects of DCVs on tumor level.

Then we consider an optimal control problem with final value of tumor mass as cost function and, as optimization horizon, the treatment period of six months. Since the possible schedules take values in a finite dimensional set, the optimal control problem reduces to an optimization ones. However, we used typical tools of optimal control to approximate the effect of DCVs and compute the gradient of the cost function with respect to the schedule. The latter is obtained via the solution of a generalized variational equation.

Simulations, run for an optimization procedure based on steepest decent, shows consinstent de- 

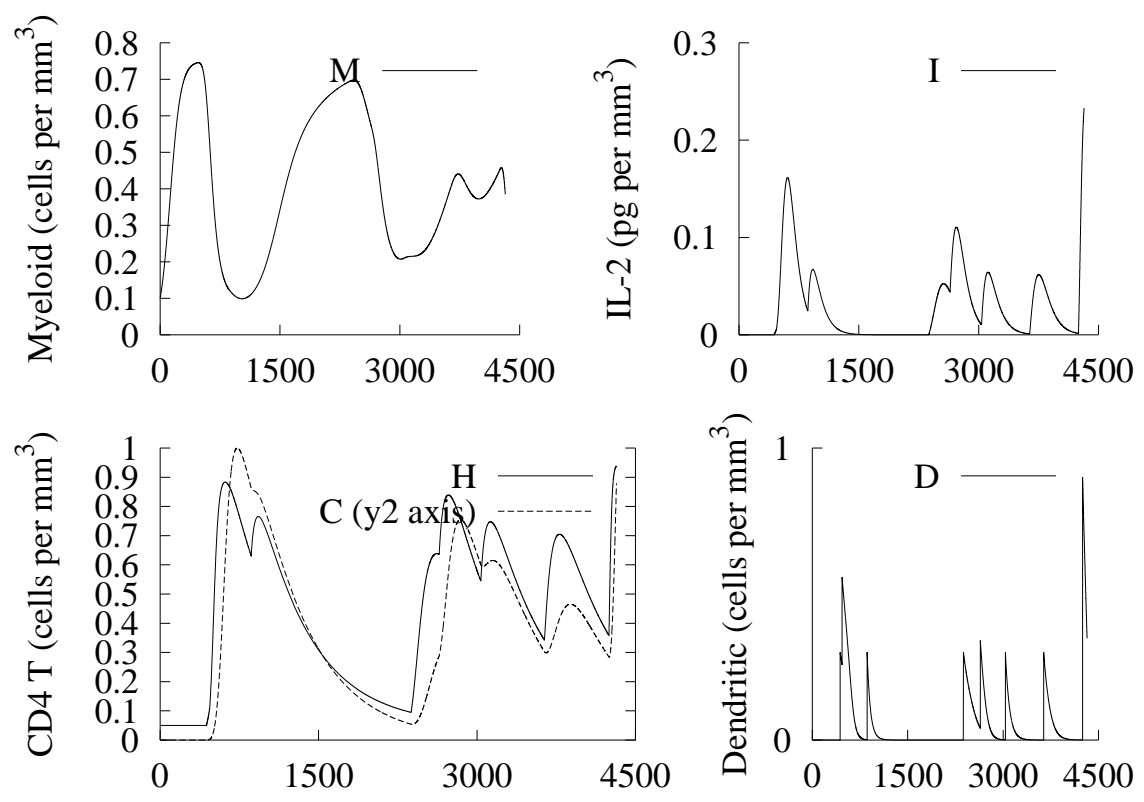

Figure 5: The dynamics of the system subjected to the optimiezed schedule of injections of DCs (figure 4 . The four plots are as in figure 1 and show peaks of immune activity after each injection and, at the same time, reduction of the tumor mass.)

crease of final tumor mass. Results are quite satisfactory, but, even if the adjoint equation is linear, there are serious numerical troubles for high number of vaccinations if one does not use a high order method. Fortunately the possibility of computing at the same time the reference trajectory and the adjoint equation permits to keep bounded the computational time and the memory needs. Further research should go in the direction of improving the model adding more cell populations and adjusting the effects of DCVs on tumor levels and other cells. Another possible direction is to consider more complicate optimal control problems. e.g. including the maximal level of the tumor during the treatment period as cost (and/or constraint) or asking for a minimal separation of vaccination times. However the former leads to optimal control and optimization problems much more difficult to be treated.

\section{References}

[1] D. Boczkowski, S. K. Nair, J. H. Nam, H. K. Lyerly, E. Gilboa, Induction of tumor immunity and cytotoxic T lymphocyte responses using dendritic cells transfected with messenger RNA amplified from tumor cells, Cancer Res. 2000 Feb 15;60(4):1028-34.

[2] Cancer Modeling and Simulation, Preziosi L. (ed.), Chapman \& Hall/CRC Press (UK), London, June 26,2003. 
[3] A. B. Dietz, M. R. Litzow, D. A. Gastineau, S. Vuk-Pavlovic, Engineering dendritic cell grafts for clinical trials in cellular immunotherapy of cancer: example of chronic myelogenous leukemia, Croat Med J. 2001 Aug;42(4):428-35.

[4] G. B. Folland, Real analysis modern techniques and their applications, Wiley and sons, 1999.

[5] M. Garavello, B. Piccoli, Hybrid necessary principle, SIAM J. Control Optim., 43, No. 5, (2005) pp. 1867-1887.

[6] R. A. Goldsby, T. J. Kindt, B. A. Osborne, Kuby Immunology, W.H. Freeman and Company, IV ed., NY (2000).

[7] A. Perelson, G. Weisbuch, Immunology for physicists, Rev. Mod. Phys. 1997; 69:1219.

[8] B. Piccoli, H. J. Sussmann, Regular synthesis and sufficiency conditions for optimality, SIAM J. Control Optim. 2000; 39:359-410.

[9] L. S. Pontryagin, V. Boltianski, R. Gamkrelidze, E. Mitchtchenko, The Mathematical Theory of Optimal Processes, John Wiley and Sons Inc., 1961.

[10] H. J. Sussmann, A nonsmooth hybrid maximum principle, in Stability and Stabilization of Nonlinear Systems, D. Aeyels, F. Lamnabhi-Lagarrigue and A. J. van der Schaft Eds., Proceedings of the First European Community Nonlinear Control Network Workshop on Stability and Stabilization, Ghent, Belgium, March 15-16, 1999, Lecture Notes in Control and Information Sciences, no. 246, Springer-Verlag, pp. 325-354. 